\title{
PLANTA DIDÁTICA SMAR PD3: AJUSTE DOS PARÂMETROS DO CONTROLADOR PI DO TANQUE DE AQUECIMENTO - Parte B
}

\author{
N. B. DOMINGOS ${ }^{1}$, C. R. R. MORAIS ${ }^{2}$, J. V. NICACIO ${ }^{3}$ e A. G. TÔRRES ${ }^{4}$ \\ ${ }^{1}$ Universidade Federal de Viçosa, Departamento de Química \\ ${ }^{2}$ Universidade Federal de Viçosa, Departamento de Química \\ ${ }^{3}$ Universidade Federal de Viçosa, Departamento de Engenharia Agrícola e Ambiental \\ ${ }^{4}$ Universidade Federal de Viçosa, Departamento de Engenharia Elétrica \\ E-mail: nathaliabdomingos@gmail.com
}

\begin{abstract}
RESUMO: $O$ controle PID (proporcional, integral e derivativo) é importante para assegurar a padronização e segurança de diversos processos químicos de uma indústria, assim como a qualidade de seus produtos. Este trabalho foi realizado no tanque de aquecimento da planta didática Smar PD3 com a sintonia do controlador PI (proporcional e integral) e a análise de estabilidade do sistema de controle. De posse das funções de

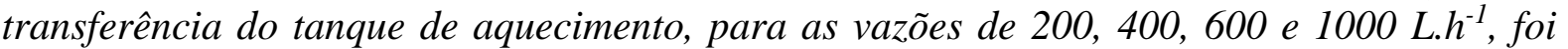
realizada a sintonia do controlador PI para a vazão de $600 \mathrm{~L}^{-1} \mathrm{~h}^{-1}$ e posterior validação do controle experimentalmente na planta, que mostrou resultados satisfatórios. Para as outras vazões foram utilizados os mesmos parâmetros do controlador encontrados para a vazão de

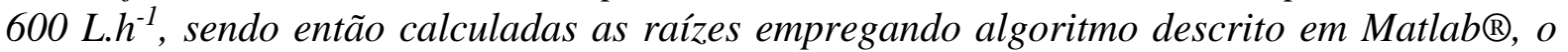
que comprovou a validade do controle para todas as vazões estudadas.
\end{abstract}

PALAVRAS-CHAVE: Processos Químicos; Controlador PI; Estabilidade.

\section{INTRODUÇÃO}

A performance requerida nas plantas de processos tem se tornado cada vez mais difícil de satisfazer. Tendo em vista o aumento da ênfase colocada na operação de plantas de forma segura e eficiente, o interesse em controle de processos se torna cada vez mais importante. Sem sistemas de controle de processos automatizados seria impossível operar plantas modernas de forma segura, rentável e ainda satisfazendo a qualidade do produto e requisitos ambientais (SEBORG, EDGAR, MELLICHAMP, 2003).

Considerando o controle Feedback, diversos tipos de controladores podem ser utilizados, porém o PID (proporcional, integral, derivativo) é o mais utilizado industrialmente. A planta Smar PD3, a qual foi utilizada neste trabalho, utiliza o controlador 
PID para controlar dois tanques, sendo um de aquecimento e outro de mistura.

Após definida a função de transferência do processo, demonstrada por Morais et al (2017), é possível fechar a malha de controle e encontrar a função de transferência global, assim como ajustar os parâmetros do controlador, sendo eles o ganho do controlador proporcional $\left(\mathrm{K}_{\mathrm{P}}\right)$ e o ganho do controlador integral $\left(\mathrm{K}_{\mathrm{I}}\right)$.

Dessa forma, o objetivo deste trabalho foi realizar o ajuste dos parâmetros do controlador PID da planta didática Smar PD3, que permitiu o controle do processo de aquecimento para diversas condições.

\section{REVISÃO DE LITERATURA}

\subsection{Planta Didática}

De acordo com informações obtidas no Manual de Instruções: Operações e Manutenção (2012) fornecido pela Smar, a configuração, operação e supervisão da Planta Didática Smar são feitas a partir de uma estação de trabalho, isto é, um microcomputador com aplicativos de softwares desenvolvidos pela Smar e específicos para cada etapa do processo industrial. Através do Sistema de Automação Industrial SYSTEM302, que utiliza a tecnologia Foudation Fieldbus, utiliza-se as ferramentas de configuração e operação para atuar nos registros dos equipamentos, modificando valores internos dos transmissores e posicionadores, e alterando os modos operacionais das malhas de controle.

\subsection{Controle Feedback}

O controlador é o elemento da malha de controle que possui "inteligência", sendo que realiza a operação de decisão do sistema, comparando o valor da variável controlada com o valor de referência ou set-point. Dessa forma, envia um sinal adequado ao elemento final de controle, que ajusta as variáveis manipuladas, com o objetivo de manter a variável controlada em seu valor desejado (STEPHANOPOULOS, 1984).

Define-se variável controlada como a grandeza que é medida e que se deseja controlar, sendo que, no controle feedback, a variável controlada é a saída do sistema (OGATA, 2010). Já o elemento final de controle é o componente da malha que implementa a decisão tomada pelo controlador. Alguns elementos finais de controle típicos podem ser válvulas, bombas ou compressores de velocidade variável, relés e resistências elétricas (STEPHANOPOULOS, 1984).

Para que se tenha um sistema de controle funcionando corretamente é necessário selecionar a ação de controle adequada para cada sistema (STEPHANOPOULOS, 1984). Segundo Ogata (2010) os controladores industriais podem ser classificados, de acordo com suas ações de controle, em controladores on-off, controladores proporcionais, controladores proporcional-integrais (PI), controladores proporcional-derivativos (PD) e controladores proporcional-integral-derivativos (PID). 
O controlador PID é o mais usado em indústrias de processos químicos por causa de sua simplicidade, robustez e fácil modelagem (JENG, TSENG, CHIU, 2014). Em adição, este tipo de controlador é de baixo custo e apresenta ótima performance em diversos sistemas de controle (SUNGTHONGA, ASSAWINCHAICHOTEB, 2016).

\subsection{Modelagem de Sistemas}

No projeto de controladores para processos químicos, é necessário o conhecimento da representação matemática dos fenômenos físicos e químicos que ocorrem em tais processos, sendo que esta representação constitui o modelo do sistema.

Informações detalhadas sobre a teoria de modelagem de sistemas, assim como a modelagem do sistema descrito neste trabalho, podem ser encontradas em Morais et al (2017).

\section{$\underline{\text { Função de transferência de segunda ordem }}$}

Como pode ser visto com mais detalhes em Morais et al (2017), as funções de transferência são utilizadas para descrever as relações de entrada e saída de um sistema.

Um sistema de segunda ordem é aquele cuja saída é modelada por uma equação diferencial de segunda ordem, sendo que sua forma padrão é:

Erro! Fonte de referência não encontrada.

Onde:

Erro! Fonte de referência não encontrada. é o tempo característico ou período natural de oscilação do sistema, o qual determina o tempo de resposta do sistema.

$\zeta$ é o fator de amortecimento, adimensional, que dá a medida da quantidade de amortecimento do sistema, ou seja, o grau de oscilação na resposta do processo após uma perturbação.

Erro! Fonte de referência não encontrada. é o ganho do sistema.

Aplicando a transformada de Laplace na Equação 1e rearranjando, tem-se:

$$
G(s)=\frac{K_{p}}{\tau^{2} s^{2}+2 \zeta \tau s+1}
$$

Onde s é uma variável real ou complexa do espaço de transformada de Laplace.

Logo, a Equação 2 é a função de transferência de um sistema de segunda ordem. 


\section{$\underline{\text { Resposta transiente de sistemas de segunda ordem }}$}

É possível se aplicar sinais de entrada em determinado processo. As perturbações em degrau são funções que modificam a resposta de um sistema de um nível ao outro até se tornar constante (LUYBEN, 1996).

Sabe-se que a transformada de Laplace de uma função degrau é dada pela Equação 3:

$$
f(s)=\frac{A}{S}
$$

Para sistemas de segunda ordem, aplicando a Equação 3 na Equação 2:

$$
y(s)=\frac{A K_{p}}{s\left(\tau^{2} s^{2}+2 \zeta \tau s+1\right)}
$$

Os dois polos da função de transferência são dados pelas raízes do polinômio característico, representado pela Equação 5:

$$
\tau^{2} s^{2}+2 \zeta \tau s+1=0
$$

Sendo que as raízes são representadas pelas Equações 6 e 7 e a resposta do sistema é dada pela Equação 8:

$$
\begin{gathered}
p_{1}=-\frac{\zeta}{\tau}+\frac{\sqrt{\zeta^{2}-1}}{\tau} \\
p_{2}=-\frac{\zeta}{\tau}-\frac{\sqrt{\zeta^{2}-1}}{\tau} \\
y(s)=\frac{A K_{p} / \tau^{2}}{s\left(s-p_{1}\right)\left(s-p_{2}\right)}
\end{gathered}
$$

A resposta após a aplicação de estímulos dependerá da localização dos dois polos da equação característica no plano complexo $\left(\mathrm{p}_{1}\right.$ e $\left.\mathrm{p}_{2}\right)$. Os três casos possíveis são:

- Quando Erro! Fonte de referência não encontrada. observa-se pelas Equações 6 e 7 a existência de dois polos reais e distintos, o que torna o sistema superamortecido, sem oscilações e com a resposta lenta.

- Quando Erro! Fonte de referência não encontrada. observa-se pelas Equações 6 e 7 a existência de dois polos reais e iguais, o que torna o sistema criticamente amortecido, sem oscilações e com resposta mais rápida quando comparada ao sistema superamortecido.

- Quando Erro! Fonte de referência não encontrada. observa-se pelas Equações 6 e 7 a existência de dois polos complexos conjugados, o que torna o sistema 
subamortecido, com oscilações e reposta rápida.

A maioria dos distúrbios em sistemas de engenharia química são distúrbios no carregamento, como variações na taxa de alimentação, composição da alimentação, pressão do vapor de aquecimento, temperatura da água de resfriamento, etc. O controlador feedback funciona retornando a variável controlada para seu set-point quando um distúrbio na entrada ocorre, através de mudanças adequadas na variável controlada. A resposta da malha fechada à variação de um distúrbio é chamada de controle regulador (LUYBEN, 1996).

Mudanças no set-point também podem ser feitas, particularmente em processos alimentados ou em mudanças de uma condição de operação a outra em processos contínuos. Essas mudanças no set-point também agem como distúrbios ao sistema de malha fechada. A função do controlador feedback é conduzir a variável controlada para corresponder ao novo set-point. A resposta em malha fechada a distúrbios no set-point é chamada de servo controle (LUYBEN, 1996).

\subsection{Sistemas de Malha Fechada}

O funcionamento de controladores, assim como do controlador PID, se baseiam no valor do erro que é calculado entre a variável controlada e seu valor desejado, o set-point. Devido a esse erro, é gerado um sinal de controle que visa eliminar o erro. A Figura 1 mostra um diagrama de blocos do controle PID e a Figura 2 mostra um diagrama de blocos genérico de uma malha de controle fechada (STEPHANOPOULOS, 1984).

A estrutura do controlador PID consiste em três valores dos parâmetros $K_{C}, K_{I}$ e $K_{D}$. É fato que quanto mais apropriados os parâmetros do PID são, mais preciso será o controle do sistema (SUNGTHONGA et al, 2016).

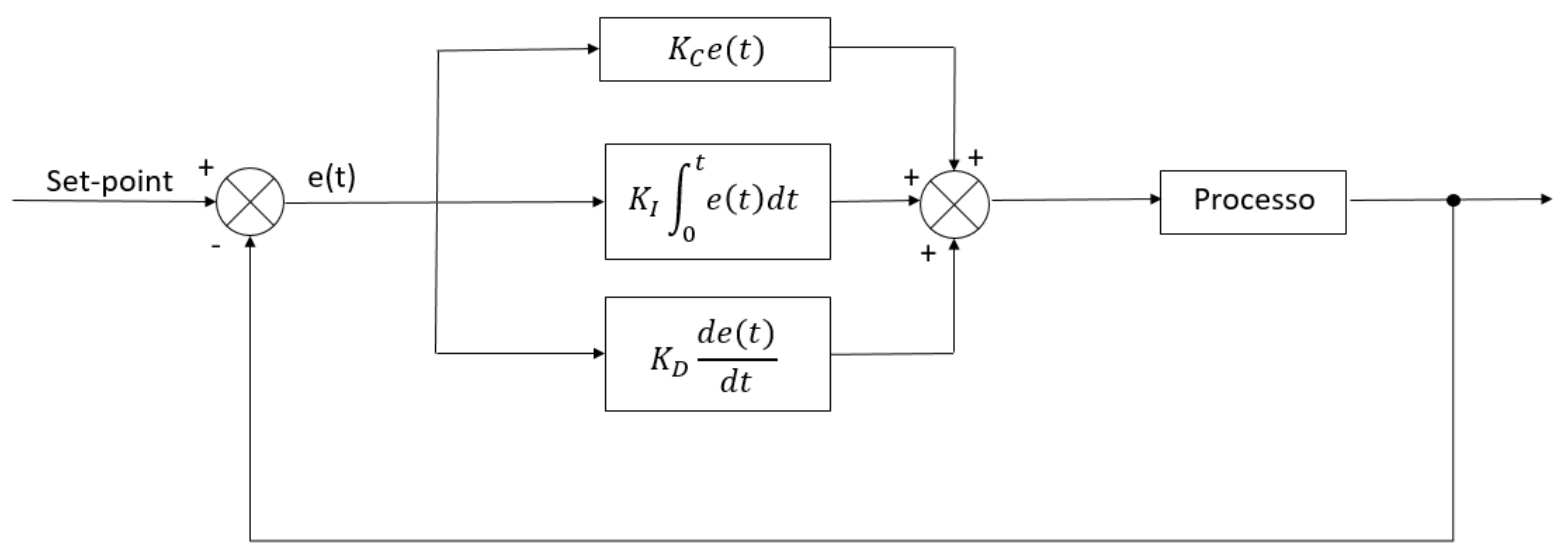

Figura 1 - Diagrama de blocos do controle PID. 


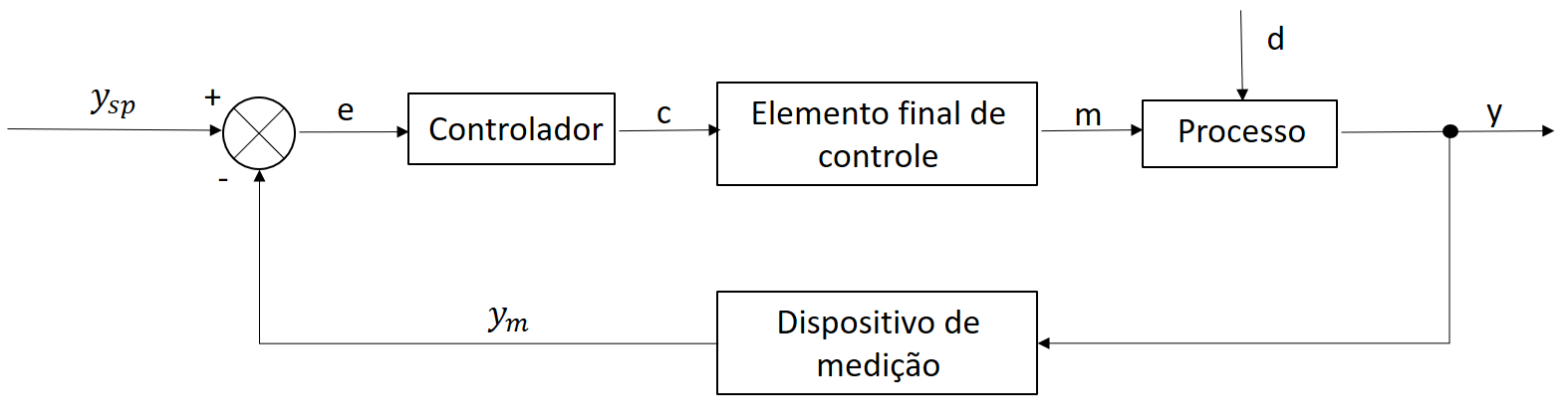

Figura 2 - Sistema de malha fechada genérico.

Sabe-se que os ganhos (K) apresentados na Figura 1 são:

Erro! Fonte de referência não encontrada.é o ganho do controlador proporcional;

Erro! Fonte de referência não encontrada. é o ganho do controlador integral;

Erro! Fonte de referência não encontrada. é o ganho do controlador derivativo.

e(t) é o erro entre a variável controlada e o set-point.

Aplicando a transformada de Laplace em cada uma das funções características do controlador PID tem-se suas funções de transferência:

Controle proporcional: Erro! Fonte de referência não encontrada.;

Controle integral: Erro! Fonte de referência não encontrada.;

Controle derivativo: Erro! Fonte de referência não encontrada..

Onde:

Erro! Fonte de referência não encontrada. é o tempo integrativo, que é o tempo para que a saída do integrador atinja o valor de Erro! Fonte de referência não encontrada. para uma entrada unitária.

Erro! Fonte de referência não encontrada. é o tempo derivativo, sendo que representa o avanço introduzido pela ação derivativa. Um aumento no parâmetro do tempo derivativo, por exemplo, faz com que o sistema de controle reaja mais fortemente às mudanças no parâmetro de erro, aumentando a velocidade da resposta global de controle do sistema.

É possível fazer um novo diagrama semelhante àquele apresentado na Figura $2 \mathrm{com}$ as respectivas funções de transferência de cada elemento. A Figura 3 mostra o diagrama de malha fechada com as funções de transferência. 


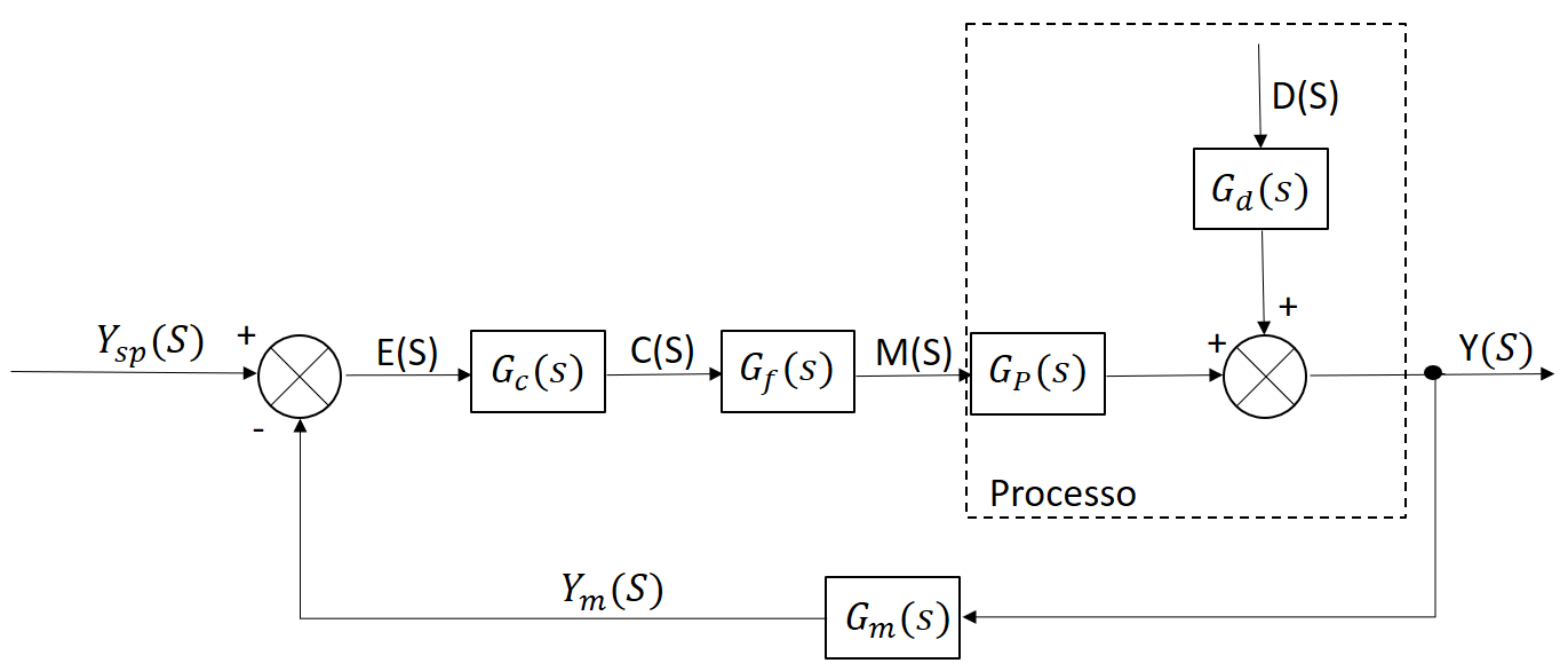

Figura 3 - Diagrama de malha fechada.

Onde:

Erro! Fonte de referência não encontrada.é a variável controlada;

Erro! Fonte de referência não encontrada. é o set-point;

Erro! Fonte de referência não encontrada. é a função de transferência do processo;

Erro! Fonte de referência não encontrada. é a função de transferência do distúrbio;

Erro! Fonte de referência não encontrada.é a variável de saída do elemento final de controle;

Erro! Fonte de referência não encontrada. é o distúrbio do sistema;

Erro! Fonte de referência não encontrada. é a variável medida;

Erro! Fonte de referência não encontrada. é a função de transferência do dispositivo de medição;

Erro! Fonte de referência não encontrada. é o erro entre a variável medida e o setpoint;

Erro! Fonte de referência não encontrada. é a variável de saída do controlador;

Erro! Fonte de referência não encontrada.é a função de transferência do controlador;

Erro! Fonte de referência não encontrada. é a função de transferência do elemento final de controle.

De acordo com Stephanopoulos (1984) pode-se escrever para cada um dos componentes do sistema (processo, dispositivo de medição, controlador e elemento final de controle) a função de transferência que relaciona sua saída com sua entrada. Em particular, se a dinâmica das linhas de transmissão for desprezada, tem-se que:

Processo: Erro! Fonte de referência não encontrada.

Dispositivo de medição: Erro! Fonte de referência não encontrada.

Mecanismo de controle: Erro! Fonte de referência não encontrada. 
Ação de controle: Erro! Fonte de referência não encontrada.

Elemento final de controle: Erro! Fonte de referência não encontrada.

É possível simplificar o diagrama da Figura 3 para encontrar a função de transferência de saída do sistema. Fazendo-se as devidas relações, sabe-se que:

$$
\begin{aligned}
& M(S)=G_{f}(S) C(S)=G_{f}(S) G_{c}(S) E(S) \\
& M(S)=G_{f}(S) G_{c}(S)\left[Y_{s p}(S)-Y_{M}(S)\right] \\
& M(S)=G_{f}(S) G_{c}(S)\left[Y_{s p}(S)-G_{M}(S) Y(S)\right]
\end{aligned}
$$

Substituindo a Equação 19 na Equação 12, tem-se que:

Erro! Fonte de referência não encontrada.

Erro! Fonte de referência não encontrada.

Onde a Equação 21 representa a resposta de malha fechada do processo.

\subsection{Estabilidade e Sintonia de Sistemas de Controle}

A equação característica de uma função de transferência é o denominador da mesma, sendo que os polos de uma função de transferência são os valores de $\mathrm{S}$ que tornam o denominador igual a zero, ou seja, são as raízes da equação característica.

A localização dos polos da equação característica de malha fechada no plano $\mathrm{S}$ determina a estabilidade de um sistema linear de malha fechada. Se qualquer um destes polos estiver no semi-plano direito de S, então, com o passar do tempo, eles darão origem ao modo dominante e a resposta transitória aumentará monotonamente ou oscilará com amplitude crescente, sendo que isso representa um sistema instável (OGATA, 2010). Dessa forma, a característica básica da resposta transiente de um sistema de malha fechada está intimamente relacionada à localização dos polos de malha fechada. Se o sistema tiver um ganho de malha variável, então as localizações dos polos de malha fechada dependem do valor do ganho de malha escolhido (OGATA, 2010).

Do ponto de vista de sintonia, em alguns sistemas um simples ajuste do ganho pode mover os polos de malha fechada até localizações desejadas. Então, o problema da sintonia pode se tornar a seleção de um valor apropriado do ganho (OGATA, 2010).

\section{$\underline{\text { Método do Lugar das Raízes }}$}

Um método simples de encontrar raízes da equação característica de uma função de transferência, conhecido como Método do Lugar das Raízes, é aquele que as raízes da 
equação característica são plotadas em função dos valores de um parâmetro do sistema. As raízes correspondentes a um particular valor deste parâmetro podem então ser localizadas no gráfico resultante. O parâmetro que geralmente é utilizado é o ganho da malha aberta (OGATA, 2010).

Utilizando o método do lugar das raízes pode-se prever os efeitos da localização dos polos de malha aberta, variando o valor do ganho ou adicionando polos e zeros de malha aberta. Dessa forma, esse método indica a maneira que os polos e zeros de malha aberta devem ser modificados para que a resposta atenda as especificações de performance de malha fechada (OGATA, 2010).

Com o método do lugar das raízes é possível analisar a estabilidade do sistema e fazer a alocação dos polos de malha fechada, com base no valor dos polos de malha aberta, para sintonizar o controlador.

\section{Método de Routh-Hurwitz}

Outro método utilizado para verificar a estabilidade de um sistema de controle é o de Routh-Hurwitz. Para se afirmar a estabilidade de um sistema contínuo é necessário que se determine se alguma das raízes da equação característica do sistema está no semi-plano direito de S (OGATA, 2010).

Considerando um polinômio característico genérico:

\section{Erro! Fonte de referência não encontrada.}

O critério de Routh-Hurwitz é uma condição necessária e suficiente para a estabilidade de um sistema. O procedimento se baseia em uma tabela derivada dos coeficientes do polinômio característico (OGATA, 2010), como mostrado na Tabela 1.

Tabela 1 - Critério de Routh-Hurwitz.

\begin{tabular}{c|cccc}
$\boldsymbol{S}^{\boldsymbol{n}}$ & $\boldsymbol{a}_{\mathbf{0}}$ & $\boldsymbol{a}_{\mathbf{2}}$ & $\boldsymbol{a}_{\mathbf{4}}$ & $\boldsymbol{a}_{\mathbf{6}}$ \\
$\boldsymbol{S}^{\mathbf{n - 1}}$ & $a_{1}$ & $a_{3}$ & $a_{5}$ & $a_{7}$ \\
$\boldsymbol{S}^{\boldsymbol{n - 2}}$ & $b_{0}$ & $b_{1}$ & $b_{2}$ & $b_{3}$ \\
$\boldsymbol{S}^{\boldsymbol{n - 3}}$ & $c_{0}$ & $c_{1}$ & $c_{2}$ & \\
$\ldots$ & $\ldots$ & $\cdots$ & $\ldots$ & \\
$\boldsymbol{S}^{\mathbf{3}}$ & $d_{0}$ & $d_{1}$ & & \\
$\boldsymbol{S}^{\mathbf{2}}$ & $e_{0}$ & $e_{1}$ & & \\
$\boldsymbol{S}^{\mathbf{1}}$ & $f_{0}$ & &
\end{tabular}


Onde:

$$
\begin{aligned}
& b_{0}=\frac{a_{1} a_{2}-a_{0} a_{3}}{a_{1}} \\
& b_{1}=\frac{a_{1} a_{4}-a_{0} a_{5}}{a_{1}} \\
& c_{0}=\frac{b_{0} a_{3}-a_{1} b_{1}}{b_{0}} \\
& c_{1}=\frac{b_{0} a_{5}-a_{1} b_{2}}{b_{0}} \\
& f_{0}=\frac{e_{0} d_{1}-d_{0} e_{1}}{e_{0}} \\
& g_{0}=\frac{f_{0} e_{1}-e_{0} 0}{f_{0}}=e_{1}
\end{aligned}
$$

Esse processo continua até completar a n-ésima linha, sendo que o arranjo completo de coeficientes é triangular (OGATA, 2010).

O critério de estabilidade de Routh-Hurwitz estabelece que o número de raízes da equação característica com parte real positiva é igual ao número de mudanças de sinal na primeira coluna da tabela. Este critério requer, portanto, que todos os elementos da primeira coluna tenham o mesmo sinal para que o sistema seja estável (OGATA, 2010).

\section{METODOLOGIA, RESULTADOS E DISCUSSÃO}

Este trabalho foi realizado no Laboratório de Pesquisa de Processos Industriais e Padrões de Movimentos e Acústica do Departamento de Engenharia Elétrica da Universidade Federal de Viçosa.

Para realização deste experimento foi utilizada a planta didática Smar PD3, representada na Figura 4. 


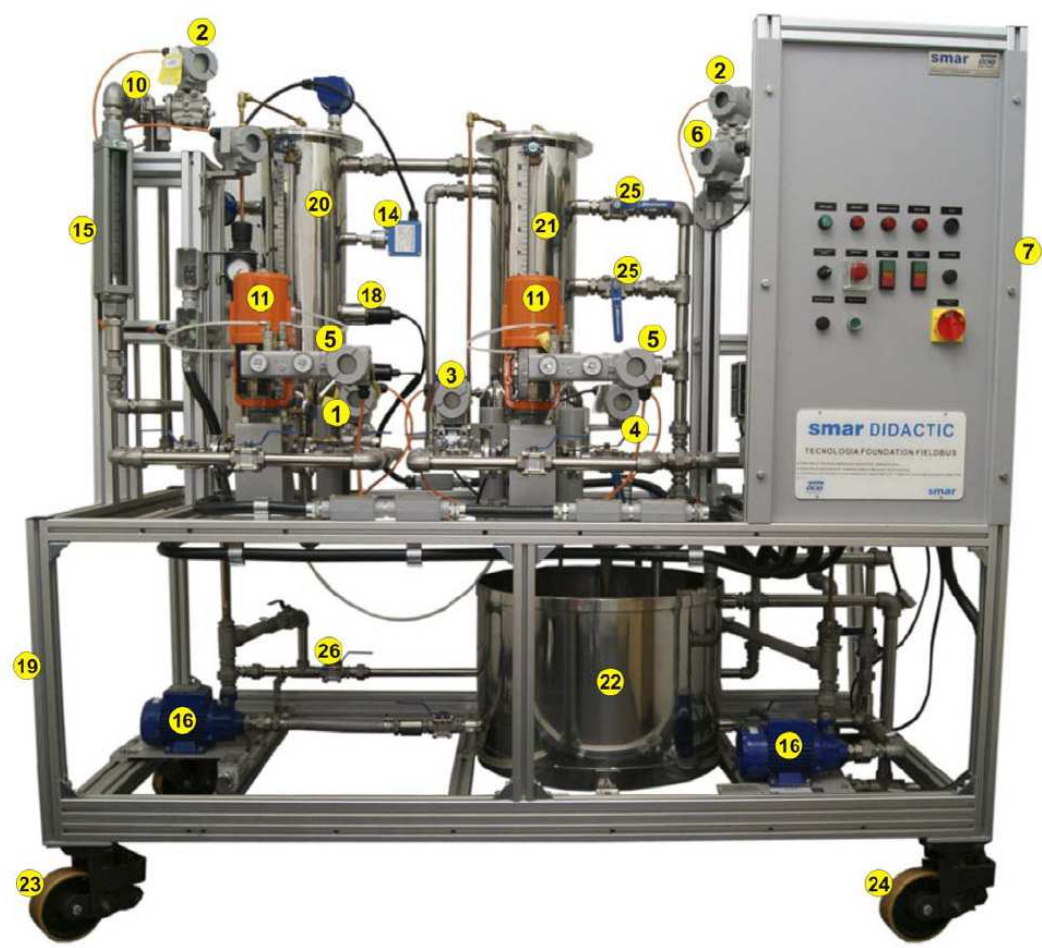

Figura 4 - Vista frontal da planta didática utilizada.

Fonte: Smar, 2012.

A água inicialmente é armazenada no tanque (22) e bombeada pela bomba (16) para o tanque de aquecimento (20). As resistências (18) aquecem a água no tanque (20), onde a vazão de entrada pode ser ajustada pela válvula (1) e observada no rotâmetro (15). A água quente que deixa o tanque de aquecimento é descartada após passar pelo tanque de mistura (21).

\subsection{Funções de Transferência de Malha Aberta}

Morais et al (2017) descreve toda a metodologia e os resultados detalhados da obtenção das funções de transferência de malha aberta do tanque de aquecimento para as vazões de

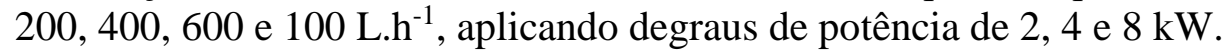

Em posse de todas as funções de transferência, foi encontrada uma função de transferência média para cada vazão aplicada, calculando uma média aritmética do ganho e da constante de tempo entre as funções de transferência encontradas para cada degrau de potência na mesma vazão. A Tabela 2 mostra os resultados.

Tabela 2 - Funções de transferência médias.

\begin{tabular}{|c|c|c|c|c|}
\hline Vazão (L/h) & 200 & 400 & 600 & 1000 \\
\hline \multirow[t]{2}{*}{ F.T. média } & 3,183 & 1,8 & 1,431 & 0,84 \\
\hline & $\overline{313 s+1}$ & $\overline{217 s+1}$ & $\overline{184 s+1}$ & $142 s+1$ \\
\hline
\end{tabular}




\subsection{Obtenção da Função de Transferência de Malha Fechada}

A Planta Smar PD3, na qual esse trabalho se baseia, possui um controlador PID, porém o componente derivativo gera muitos ruídos à resposta do sistema, o que é característico do controle derivativo. Dessa forma, o controlador utilizado nos experimentos foi o proporcional integral (PI).

Para obter o modelo do sistema de controle é necessário fechar a malha do sistema, considerando que o controlador utilizado é PI. A Equação 21 mostra a resposta genérica de uma malha fechada. Para o sistema estudado tem-se que:

$$
\begin{aligned}
& G_{p}(S)=\frac{K_{p}}{\tau_{p} S+1} \\
& G_{c}(S)=K_{c}\left(1+\frac{1}{\tau_{l} S}\right) \\
& G_{m}(S)=1 \\
& G_{f}(S)=1
\end{aligned}
$$

Onde:

$\tau_{p}$ é a constante de tempo do processo;

$K_{C}$ é o ganho do processo.

Fazendo as devidas simplificações na Equação 21 obtém-se:

$$
Y(S)=\frac{G_{p}(S) G_{c}(S)}{1+G_{p}(S) G_{c}(S)} Y_{S p}(S)+\frac{G_{d}(S)}{1+G_{p}(S) G_{c}(S)} D(S)
$$

Substituindo as Equações 29, 30, 31 e 32 em 33 e rearranjando a equação tem-se a Equação 34.

\section{ERRO! FONTE DE REFERÊNCIA NÃO ENCONTRADA.}

Como a função de transferência de malha aberta varia para cada valor de vazão, a função de transferência de malha fechada também irá mudar.

\subsection{Sintonia do Controlador PI}

Para fazer a sintonia do controlador PI deve-se encontrar os valores de Erro! Fonte de referência não encontrada. e de Erro! Fonte de referência não encontrada. que tornam o sistema estável e com uma resposta dinâmica, ou seja, que alcança o set-point rapidamente. A metodologia escolhida para tal objetivo foi o método do lugar das raízes, 
onde é feita a alocação dos polos que leva à reposta desejada.

O método do lugar das raízes sugere que o polo de malha fechada escolhido inicialmente seja o polo da função de transferência de malha aberta. O segundo polo escolhido foi dez vezes maior do que o primeiro.

A sintonia foi feita com base na função de transferência de malha aberta na vazão de 600 L.h ${ }^{-1}$, já que essa é uma vazão intermediária dos testes realizados, da seguinte forma:

A função de transferência do controlador $\mathrm{G}_{\mathrm{C}}$ é dada pela Equação 35, a qual é derivada da Equação 30.

$$
G_{C}=\frac{K i}{S}+K c
$$

E utilizando a Equação 33, para analisar apenas o efeito do controlador, considera-se a malha fechada, dada pela Equação 36.

$$
T(S)=\frac{G_{P} G_{C}}{1+G_{p} G_{C}} T_{s p}(S)
$$

Substituindo as Equações 29 e 35 na Equação 36 e simplificando para se obter uma função de transferência com o formato da Equação 34, tem-se a Equação 37 :

$$
T(S)=\frac{1+\frac{K c}{K i} S}{\frac{\tau_{p}}{K p K i} S^{2}+\frac{(K p K c+1)}{K p K i} S+1} T_{s p}(S)
$$

Para sintonizar o ganho do controlador para a vazão de $600 \mathrm{~L} \cdot \mathrm{h}^{-1}$, usou-se os polos da malha aberta da função de transferência média, que foi apresentada na Tabela 2 para esta vazão.

Sabe-se que a equação característica da função de transferência citada é representada pela Equação 38, a qual também representa a obtenção de sua raiz.

Erro! Fonte de referência não encontrada.

O polo desta função é -Erro! Fonte de referência não encontrada.. Neste método, escolhe-se o segundo polo que seja dez vezes maior que o polo da malha aberta, sendo ele então igual a -Erro! Fonte de referência não encontrada.. Assim, o polinômio que apresenta essas duas raízes é mostrado abaixo:

$$
\left(S+\frac{1}{184}\right)\left(S+\frac{1}{18,4}\right)
$$

Manipula-se matematicamente para que ele fique na forma de uma equação 
característica de segundo grau e iguala-se a equação característica da função de transferência de malha fechada para a vazão em questão. Assim, tem-se a Equação 39.

$$
3385,6 S^{2}+3385,6\left(\frac{1}{18,4}+\frac{1}{184}\right) S+1=\frac{\tau_{p}}{K p K i} S^{2}+\frac{(K p K c+1)}{K p K i} S+1
$$

Onde, tendo como base a Tabela 2 e a Equação 29:

$$
\begin{aligned}
& \tau_{p}=184 \\
& K_{p}=1,43
\end{aligned}
$$

Dessa forma, obtém-se as Equações 40 e 41.

$$
\begin{aligned}
& \frac{\tau_{P}}{K p K i}=3385,6 \rightarrow K i=0,038 \\
& \frac{(K p K C+1)}{K p K i}=3385,6\left(\frac{1}{18,4}+\frac{1}{184}\right) \rightarrow K c=6,99
\end{aligned}
$$

Sabendo-se que:.

$$
\tau_{I}=\frac{K_{c}}{K_{l}}=184,21
$$

Dessa forma, a função de transferência de malha fechada para a vazão de $600 \mathrm{~L} \cdot \mathrm{h}^{-1}$ será dada pela Equação 43.

\section{Erro! Fonte de referência não encontrada.}

Tendo como base a Equação 2, que mostra a função de transferência característica de segunda ordem, sabe-se que, da Equação 43:

$$
\begin{aligned}
& \tau=58,2 s \\
& \zeta=1,74
\end{aligned}
$$

Estes valores de Erro! Fonte de referência não encontrada. e de Erro! Fonte de referência não encontrada. foram colocados no software do controlador PI da planta Smar PD3, junto com o set-point. A temperatura inicial da água era aproximadamente de $24,6^{\circ} \mathrm{C}$ e o set-point foi ajustado para $33^{\circ} \mathrm{C}$. A Figura 5 mostra o gráfico da temperatura da água no tanque pelo tempo. 


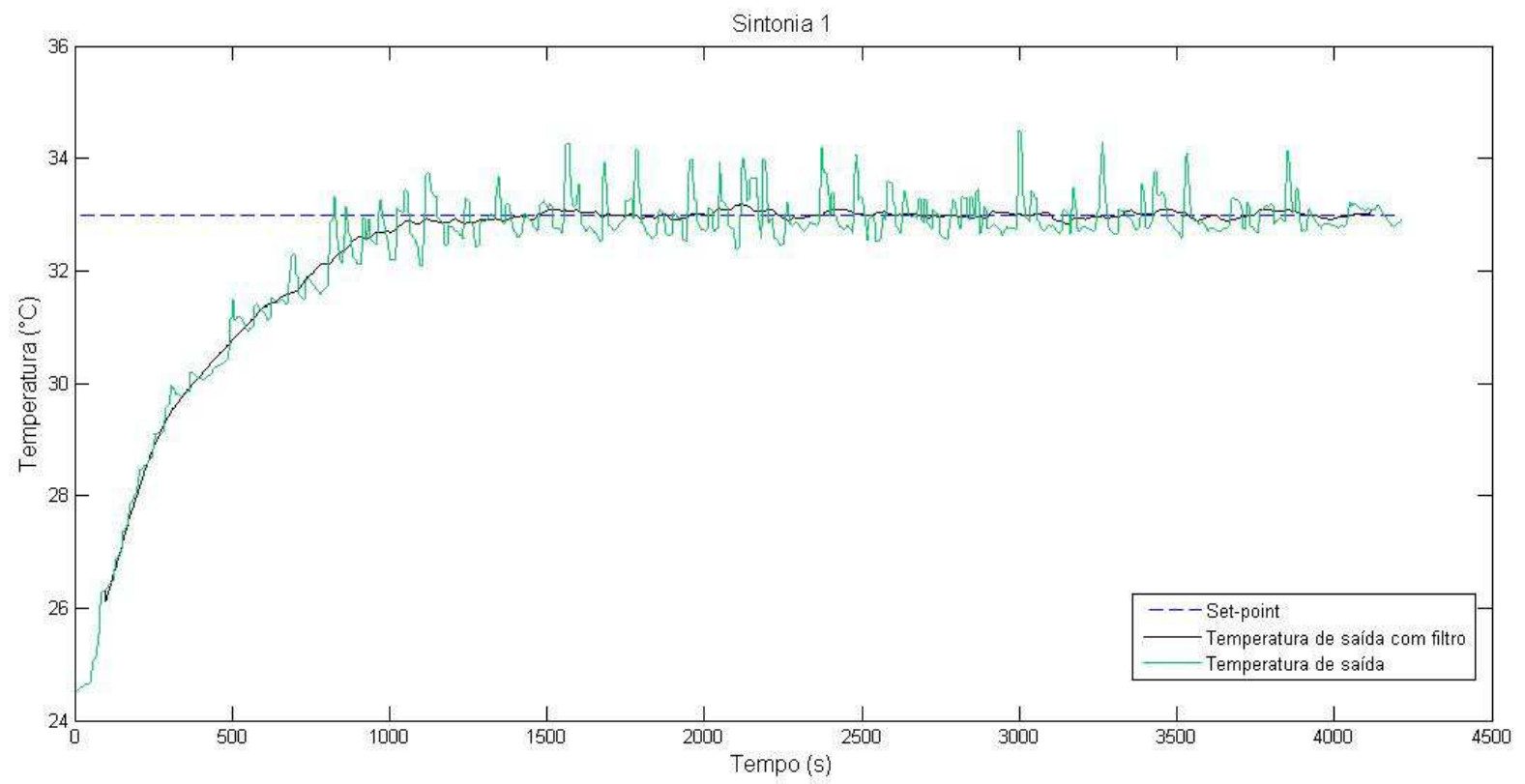

Figura 5 - Gráfico da resposta do sistema com o controle PI (Sintonia 1).

Devido a ocorrência de muitos ruídos na resposta do sistema, provenientes de distúrbios de natureza desconhecida, foi necessário o tratamento dos dados de resposta da planta ao degrau para a análise do comportamento da temperatura no tanque, utilizando a média móvel no Matlab. Este tratamento atenua as oscilações da curva sem descaracterizar o comportamento do processo, permitindo uma análise mais precisa.

É possível observar na curva com utilização do filtro na Figura 5 que o sistema é estável, além de não haver overshoot, o que mostra que o sistema é superamortecido. Além disso, a resposta do sistema é mais lenta, já que entra em regime permanente com aproximadamente 23 minutos.

Com objetivo de observar a reposta do sistema para diferentes valores de polo da malha fechada, foram feitos mais dois testes com diferentes valores de Erro! Fonte de referência não encontrada., também utilizando o filtro de média móvel para tratar os dados.

A Figura 6 mostra o teste realizado com Erro! Fonte de referência não encontrada., que são valores cem vezes menores do que aqueles utilizados no primeiro teste (Figura 5), e um set-point de $34^{\circ} \mathrm{C}$. 


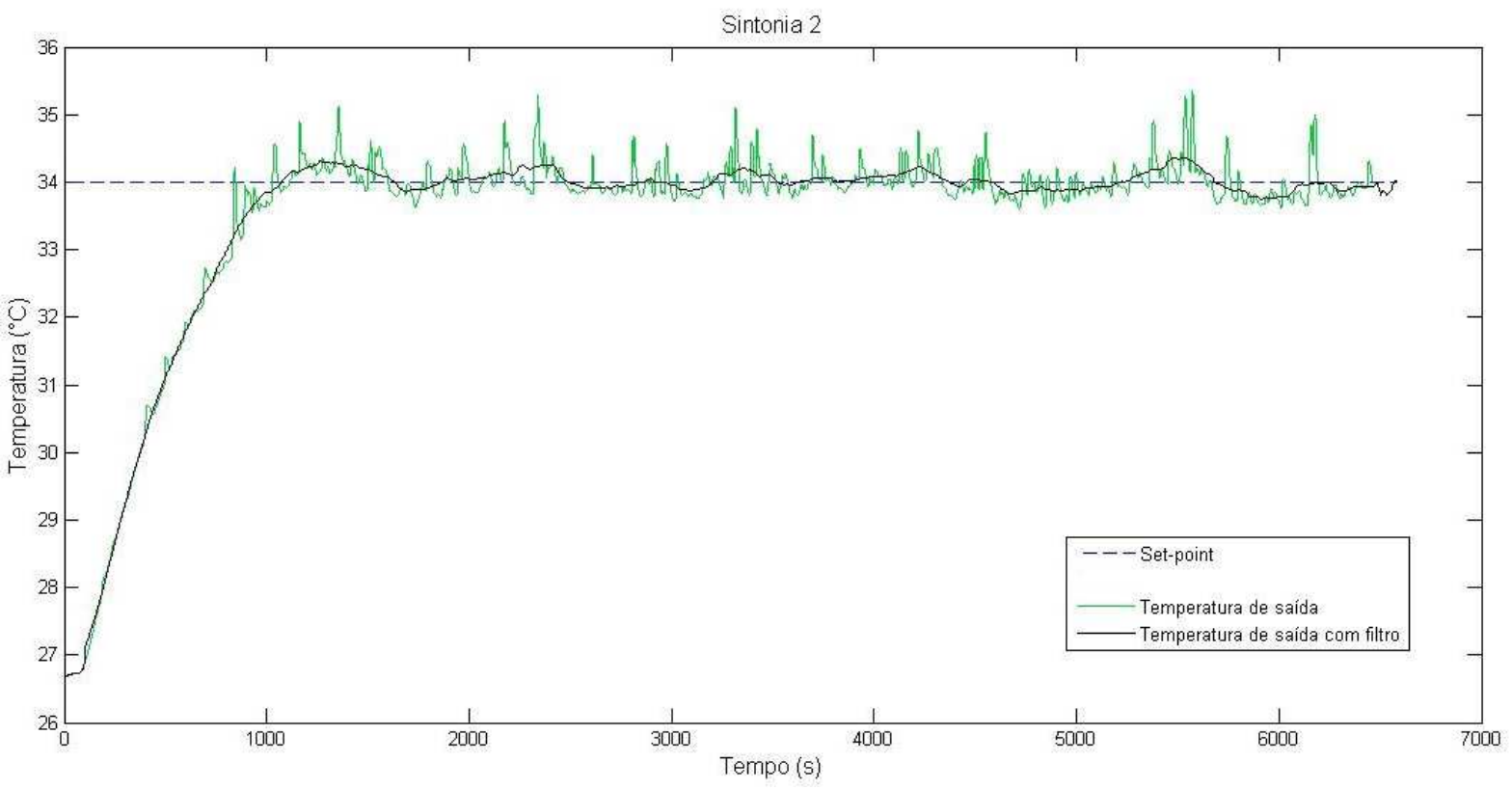

Figura 6 - Gráfico da resposta do sistema com o controle PI (Sintonia 2).

Para se observar a influência do valor do ganho do controlador na resposta do sistema, o terceiro teste foi realizado com Erro! Fonte de referência não encontrada. e um set-point de $31,6^{\circ} \mathrm{C}$, onde o valor do ganho do controlador foi duas vezes maior do que no segundo teste e o tempo integrativo foi mantido. O resultado é mostrado na Figura 7.

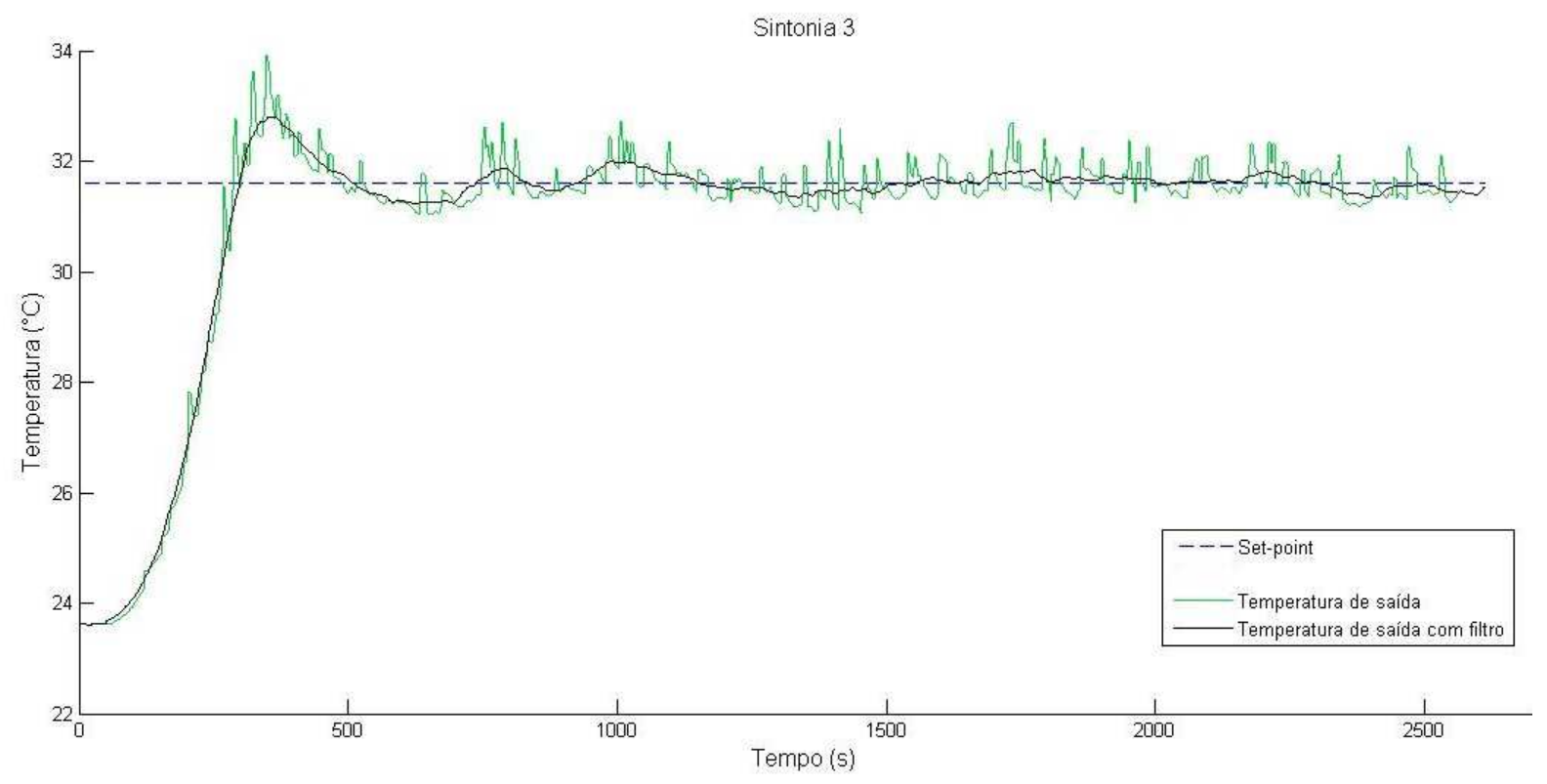

Figura 7 - Gráfico da resposta do sistema com o controle PI (Sintonia 3).

Como pode ser observado nas Figuras 6 e 7, as respostas do sistema apresentam um overshoot e são oscilatórias. Isso acontece porque ambas as funções de transferência de malha fechada apresentam polos com parte imaginária, o que gera oscilações na resposta, o que é comprovado com a análise de estabilidade e do lugar das raízes do sistema. A sintonia 
2, da Figura 6, possui um menor overshoot quando comparada a sintonia 3 (Figura 7).

Analisando a estabilidade do sistema pelo critério de Routh-Hurwitz:

\begin{tabular}{c|c}
$\boldsymbol{S}^{\mathbf{2}}$ & $\frac{184 \tau_{I}}{1,43 K_{c}}$ \\
$\boldsymbol{S}^{\mathbf{1}}$ & $\frac{\tau_{I}}{1,43 K_{c}}+\tau_{l}$ \\
$\boldsymbol{S}^{\mathbf{0}}$ & 1
\end{tabular}

Para estabilidade sabe-se que:

$$
\begin{aligned}
& \frac{184 \tau_{I}}{1,43 K_{c}}>0 \rightarrow \tau_{I}>0 \\
& \frac{\tau_{I}}{1,43 K_{c}}+\tau_{I}>0 \rightarrow K_{c}>-0,7
\end{aligned}
$$

Dessa forma, observa-se que o sistema é estável para qualquer valor de Erro! Fonte de referência não encontrada.e Erro! Fonte de referência não encontrada. considerado neste trabalho. As oscilações observadas nos gráficos das Figuras 6 e 7 podem ser explicadas pela análise do valor de fator de amortecimento de cada função de transferência de malha fechada.

Pela Equação 34 e Equação 5, para vazão de 600L/h, observa-se que:

$$
\begin{aligned}
& \tau^{2}=\frac{\tau_{p} \tau_{I}}{K_{p} K_{c}}=\frac{184 \tau_{I}}{1,43 K_{c}} \rightarrow \tau=\sqrt{\frac{184 \tau_{I}}{1,43 K_{c}}} \\
& 2 \zeta \tau=\frac{\tau_{l}}{K_{p} K_{c}}+\tau_{I} \rightarrow \zeta=\frac{\tau_{I}+1,43 K_{c} \tau_{I}}{2 \sqrt{\left(1,43 K_{c}\right)\left(184 \tau_{I}\right)}}
\end{aligned}
$$

Sabe-se que para o sistema ser criticamente amortecido, Erro! Fonte de referência não encontrada. Então é possível obter a Equação 47 e, ao simplifica-la, obtém-se a Equação 48.

$$
\begin{gathered}
\frac{\tau_{I}+1,43 K_{c} \tau_{I}}{2 \sqrt{\left(1,43 K_{c}\right)\left(184 \tau_{I}\right)}}=1 \\
\tau_{I}=\frac{1052,48 K_{c}}{1+2,86 K_{c}+2,045 K_{c}^{2}}
\end{gathered}
$$

Assim, para o Erro! Fonte de referência não encontrada. utilizado na Sintonia 2, 
representada pela Figura 6, observa-se, pela Equação 48, que Erro! Fonte de referência não encontrada.deveria ser maior ou igual a 60,88 para que o sistema não oscilasse. Da mesma forma, para a Sintonia 3, representada na Figura 7, Erro! Fonte de referência não encontrada. deveria ser maior ou igual a 102,29 com um Erro! Fonte de referência não encontrada. Como em ambos os casos o Erro! Fonte de referência não encontrada. utilizado foi de 1,84, comprova-se a oscilação observada.

O gráfico do lugar das raízes mostra todos os valores das raízes do sistema, variando o ganho de zero ao infinito, sendo que quando existem raízes no semi plano esquerdo de $\mathrm{S}$ (parte real positiva) o sistema é instável. O gráfico também mostra quando existem raízes imaginárias, o que está associado com oscilações que podem ser observadas no sistema. Neste gráfico, o eixo de valores reais das raízes é representado na horizontal, enquanto a vertical representa o eixo imaginário.

O gráfico do lugar das raízes para as sintonias 2 e 3 é mostrado na Figura 8.

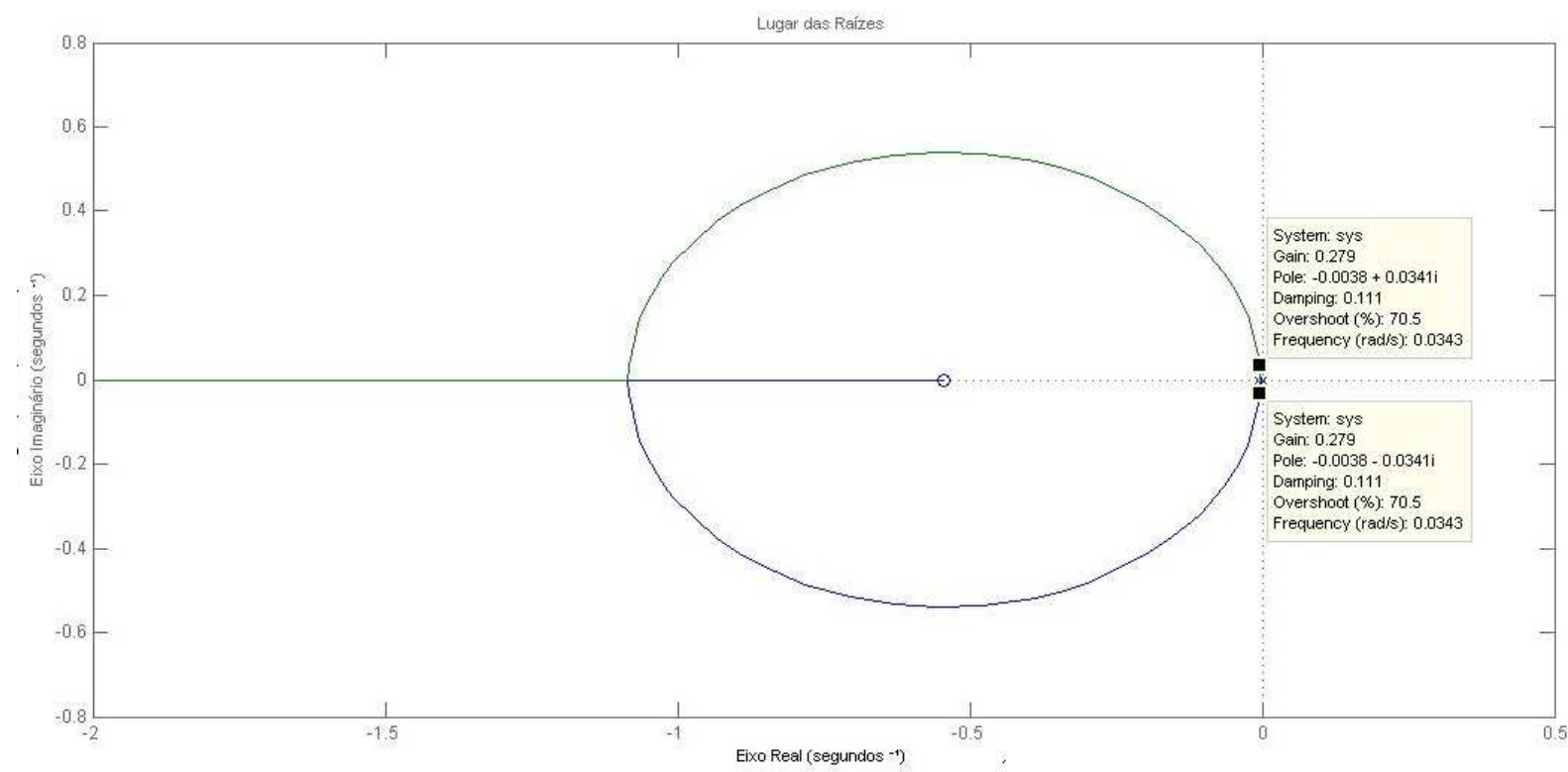

Figura 8 - Lugar das raízes para equação característica das sintonias 2 e 3.

Para o valor de Kc utilizado na Sintonia 1, o valor de Erro! Fonte de referência não encontrada. mínimo para que não houvesse oscilação era de 60,77. Como foi utilizado Erro! Fonte de referência não encontrada., não foi observada nenhuma oscilação do sistema, como pode ser observado na reposta da Figura 5. Esta estabilidade, associada a não existência de oscilações, pode ser comprovada pelo gráfico do lugar das raízes, na Figura 9, já que não existem raízes imaginárias. 


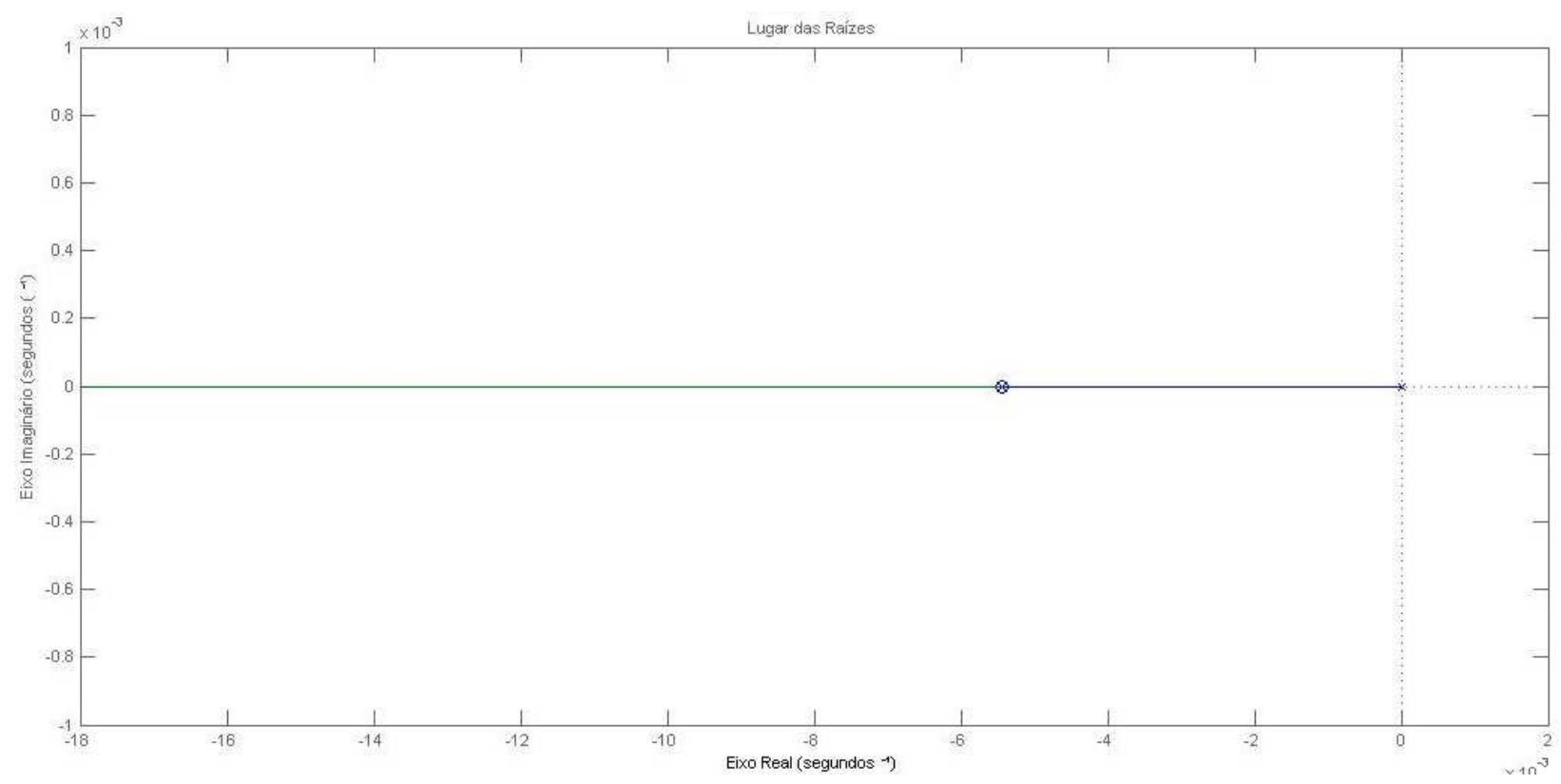

Figura 9 - Lugar das raízes da equação característica da sintonia 1.

Como já era esperado, a resposta obtida com a sintonia 1 (Figura 5) é mais lenta do que as respostas das sintonias 2 e 3 (Figuras 6 e 7), tendo em vista que o sistema da primeira é superamortecida e os dois últimos são subamortecidos.

Com o objetivo de investigar a validade do controle obtido anteriormente para as outras

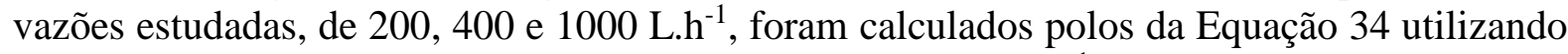
os mesmos parâmetros do controlador para a vazão de $600 \mathrm{~L} \cdot \mathrm{h}^{-1}, \mathrm{~K}_{\mathrm{c}}=7,0$ e $\mathrm{K}_{\mathrm{I}}=0,038$, e os parâmetros das funções de transferência localizadas na Tabela 2.

As Figuras 10, 11 e 12 mostram os polos de malha fechada para as vazões de 200, 400 e 1000 L.h. ${ }^{-1}$, respectivamente. 


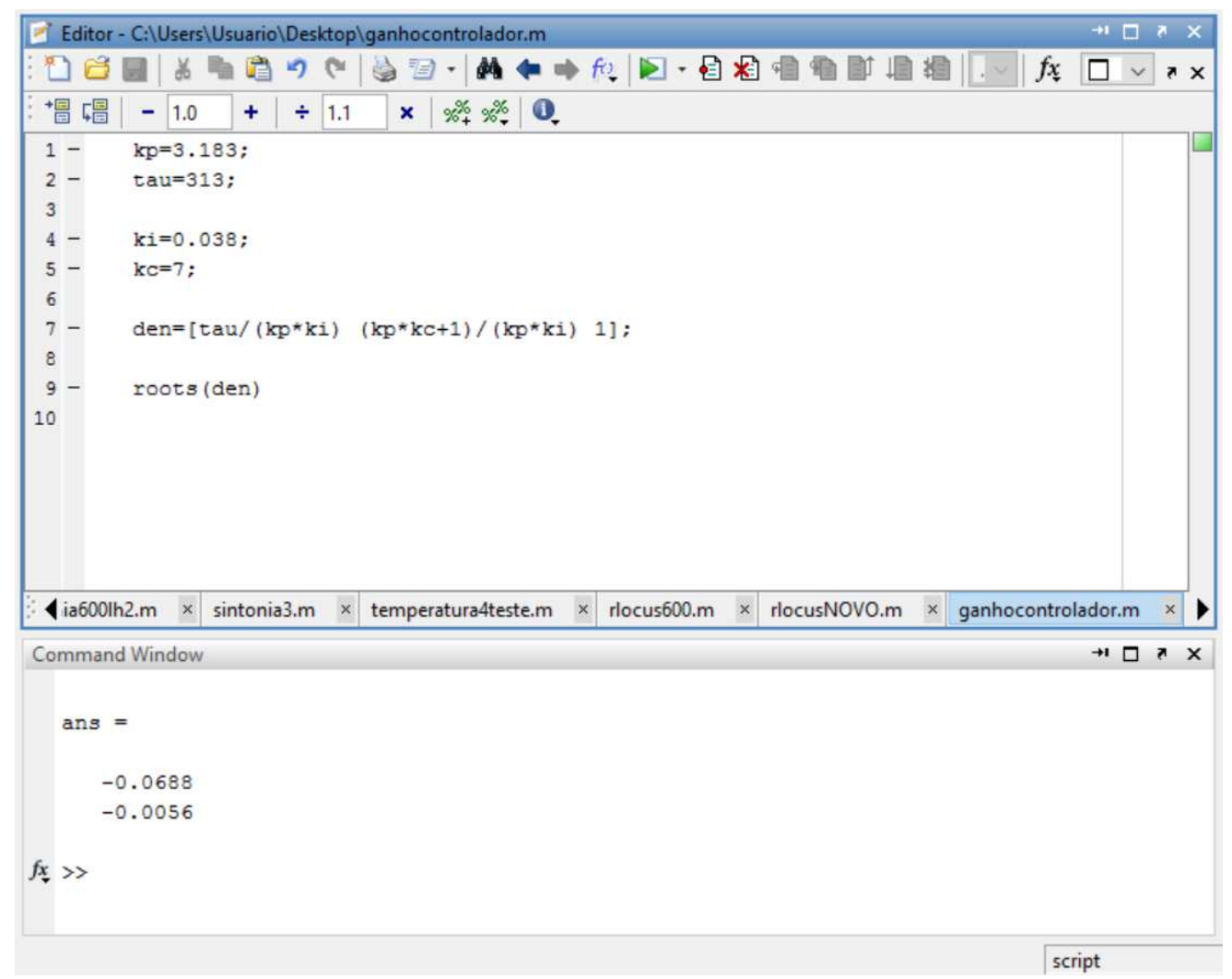

Figura 10 - Polos para vazão de 200 L/h.

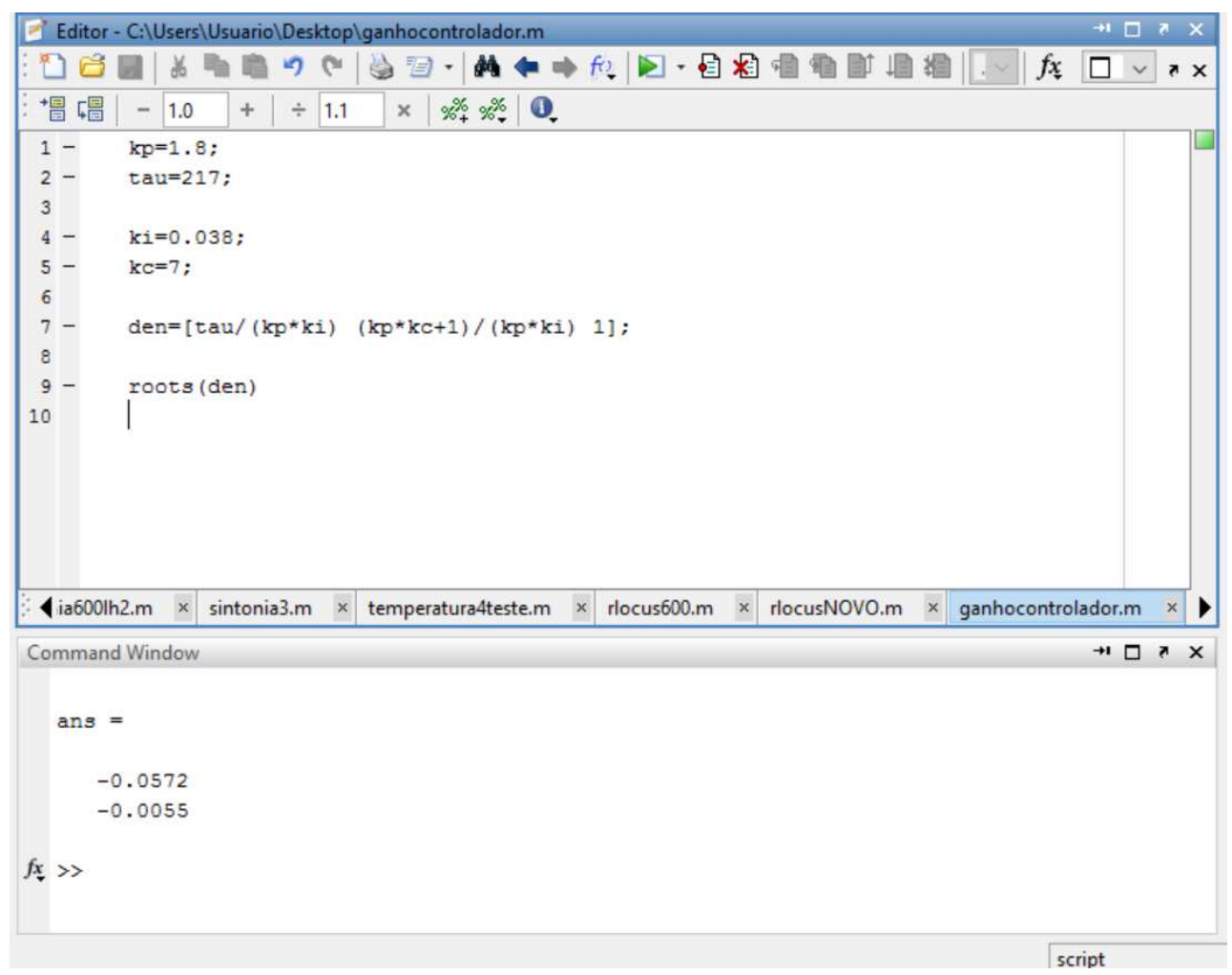

Figura 11 - Polos para vazão de 400 L/h. 


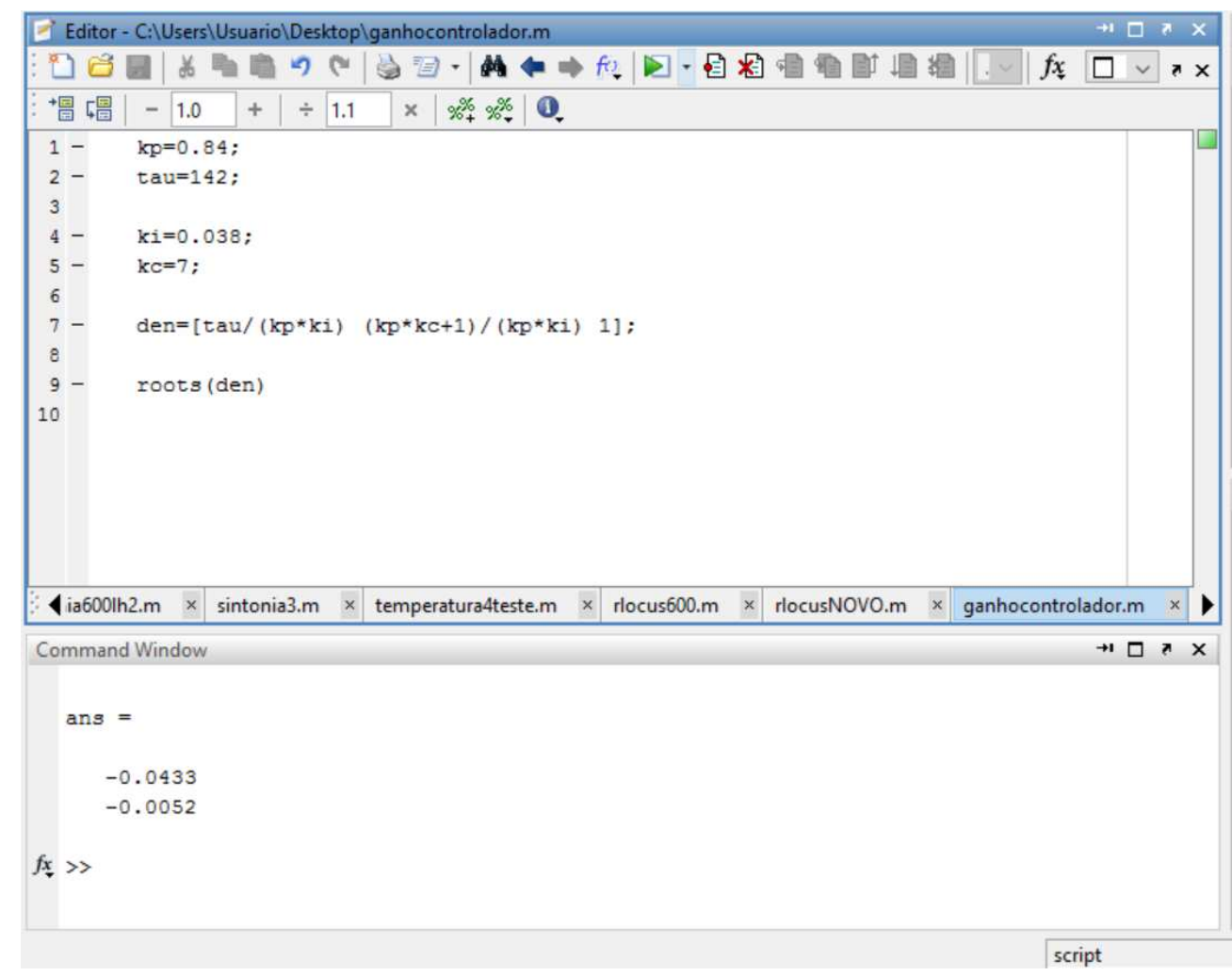

Figura 12 - Polos para vazão de 1000 L/h.

Como pode ser observado nas Figuras 10, 11 e 12 os polos de malha fechada para todas as vazões estudadas foram reais e, portanto, não apresentam oscilação, já que o sistema é superamortecido.

\section{CONSIDERAÇÕES FINAIS}

Em função dos testes realizados e dos resultados obtidos pode-se concluir que o sistema de controle projetado se mostrou eficiente para controlar adequadamente o processo de aquecimento da água, sendo que quando se deseja um processo sem oscilações a sintonia 1 (Figura 5) é mais adequada e quando se deseja uma resposta mais rápida as sintonias 2 e 3 (Figuras 6 e 7) são mais adequadas.

São propostas as seguintes sugestões para trabalhos futuros:

- Fazer o controle de vazão no tanque de aquecimento;

- Realizar a modelagem para o tanque de mistura;

- Fazer o controle de vazão no tanque de mistura, finalizando, assim, o controle completo da planta didática. 


\section{REFERÊNCIAS}

JENG, J.; TSENG, W.; CHIU, M. A one-step tuning method for PID controllers with robustness specification using plant step-response data. Chemical engineering research and design 92, 545-558, 2014. Disponível em: <http://www.sciencedirect.com/science/article/pii/S0263876213003948>. Acesso em 05 set. 2016.

KWONG, W. H. Introdução ao Controle de Processos Químicos com MATLAB. Volume 1. São Carlos: EdUFScar, 2012. 212p.

LUYBEN, W. L. Process Modeling, Simulation, and Control for Chemical Engineers. 2. ed. United Stated: McGraw-Hill, 1996.

MORAIS, C. R. R.; DOMINGOS, N. B.; NICACIO, J. V.; TÔRRES, A.G. Planta Didática Smar PD3: Modelagem e Simulação do Tanque de Aquecimento - Parte A. The Journal of Engineering and Exact Sciences - JCEC, 2017,(in press).

OGATA, K. Modern Control Engineering. 5. ed. New Jersey: Prentice Hall, 2010. 894p.

SEBORG, D. A.; EDGAR, T. F.; MELLICHAMP, D. A. Process Dynamics and Control. 2. ed. United States: John Wiley \& Sons, 2003.

SMAR - PD3 - F. Manual de Instruções: Operação e Manutenção - Plantas Didáticas. 2012. Disponívem em: < http://www.smar.com/brasil/produto/pd3-planta-didatica-hartfoundation-fieldbus-e-profibus $>$. Acesso em 15 ago. 2016.

STEPHANOPOUlOS, G. Chemical Process Control: An Introduction to Theory and Practice. New Jersey: Prentice Hall, 1984. 696p.

SUNGTHONGA, A.; ASSAWINCHAICHOTEB, W. Particle Swam Optimization Based Optimal PID Parameters for Air Heater Temperature Control System. International Electrical Engineering Congress, iEECON2016, 2-4 March 2016, Chiang Mai, Thailand. Disponível em: < http://www.sciencedirect.com/science/article/pii/S1877050916303635>. Acesso em 05 set. 2016. 


\title{
DIDACTIC PLANT SMAR PD3: ADJUSTMENT OF THE PI CONTROLLER PARAMETERS OF A HEATING TANK - Part B
}

\author{
N. B. DOMINGOS ${ }^{1}$, C. R. R. MORAIS ${ }^{2}$, J. V. NICACIO ${ }^{3}$ e A. G. TÔRRES ${ }^{4}$ \\ ${ }^{1}$ Universidade Federal de Viçosa, Departamento de Química \\ ${ }^{2}$ Universidade Federal de Viçosa, Departamento de Química \\ ${ }^{3}$ Universidade Federal de Viçosa, Departamento de Engenharia Agrícola e Ambiental \\ ${ }^{4}$ Universidade Federal de Viçosa, Departamento de Engenharia Elétrica \\ E-mail: nathaliabdomingos@gmail.com
}

\begin{abstract}
PID control (proportional, integral and derivative) is important to ensure the standardization and safety of various chemical processes in an industry, as well as the quality of its products. This work was carried out in the heating tank of the Smar PD3 didactic plant with the tuning of the PI controller (proportional and integral) and the stability analysis of the control system. With the transfer function of the heating tank for the flow rates of 200, 400, 600 and $1000 \mathrm{~L}^{-1}{ }^{-1}$, the PI controller was tuned for the flow rate of $600 \mathrm{Lh}^{-1}$ and it was done the validation of the control experimentally in the plant, which showed satisfactory results. For the other flows, the same parameters of the controller were used for the flow of $600 \mathrm{~L} \cdot \mathrm{h}^{-1}$, and the roots were calculated by with a program in Matlab ${ }^{\circledR}$, which proved the validity of the control for all the flows studied.
\end{abstract}

KEYWORDS: Chemical Processes; PI Controller; Stability. 